\title{
ALMOST EVERYWHERE CONVERGENCE OF A SUBSEQUENCE OF THE NÖRLUND LOGARITHMIC MEANS OF WALSH-KACZMARZ-FOURIER SERIES
}

\author{
KÁROLY NAGY
}

\begin{abstract}
The main aim of this paper is to prove that the maximal operator of a subsequence of the (one-dimensional) logarithmic means of Walsh-Kaczmarz-Fourier series is of weak type $(1,1)$. Moreover, we prove that the maximal operator of the logarithmic means of quadratical partial sums of double Walsh-Kaczmarz-Fourier series is of weak type $(1,1)$, provided that the supremum in the maximal operator is taken over special indices. The set of Walsh-Kaczmarz polynomials is dense in $L^{1}$, so by the well-known density argument the logarithmic means $t_{2^{n}}^{K}(f)$ converge a.e. to $f$ for all integrable function $f$.
\end{abstract}

Mathematics subject classification (2000): 42C10.

Keywords and phrases: Walsh group, Walsh-Kaczmarz system, double Walsh-Kaczmarz-Fourier series, logarithmic means, a.e. convergence.

\section{REFERENCES}

[1] G.H. Agaev, N.Ja. Vilenkin, G.M. Dzhafarli, And A.I. Rubinstein, Multiplicative systems of functions and harmonic analysis on 0-dimensional groups (Russian), Izd. ("ELM"), Baku, 1981.

[2] I. Blahota, G. Gát, Norm summability of Nörlund logarithmic means on unbounded Vilenkin groups, Anal. in Theory and Appl., 24, 1 (2008), 1-17.

[3] G. GÁt, On $(C, 1)$ summability of integrable functions with respect to the Walsh-Kaczmarz system, Studia Math., 130, 2 (1998), 135-148.

[4] G. GÁT, Investigations of certain operators with respect to the Vilenkin system, Acta Math. Hung., 61, 1-2 (1993), 131-149.

[5] G. GÁT, U. GoginaVA, Uniform and L-convergence of logarithmic means of Walsh-Fourier series, Acta Math. Sinica, English Series, 22, 2 (2006), 497-506.

[6] G. GÁt, U. GoginaVA, Almost everywhere convergence of a subsequence of the logarithmic means of quadratical partial sums of double Walsh-Fourier series, Publ. Math. Debrecen, 71, 1-2 (2007), 173-184.

[7] G. GÁt, R. TOLEDO, $L^{p}$-norm convergence of series in compact, totally disconnected groups, Analysis Mathematica, 22 (1996), 13-24.

[8] U. Goginava, Almost everywhere convergence of subsequence of logarithmic means of WalshFourier series, Acta Math. Acad. Paed. Nyíregyháziensis, 21 (2005), 169-175.

[9] F. Móricz, F. SCHIPP, W.R. WAdE, Cesáro summability of double Walsh-Fourier series, Trans. Amer. Math. Soc., 329 (1992), 131-140.

[10] F. MórICZ, A. SidDIQI, Approximation by Nörlund means of Walsh-Fourier series, Journal of Approx. Theory, 70, 3 (1992), 375-389.

[11] K. Nagy, On the two-dimensional Marcinkiewicz means with respect to Walsh-Kaczmarz system, Journal of Approx. Theory, 142 (2006), 138-165.

[12] F. Schipp, W. R. Wade, P. Simon, And J. PÁL, Walsh Series. An Introduction to Dyadic Harmonic Analysis, Adam Hilger, Bristol-New York, 1990.

[13] P. Simon, Strong convergence of certain means with respect to the Walsh-Fourier series, Acta Math. Hung., 49 (1987), 425-431. 
[14] P. Simon, $(C, \alpha)$ summability of Walsh-Kaczmarz-Fourier series, J. of Approx. Theory, 127 (2004), $39-60$.

[15] V.A. Skvortsov, On Fourier series with respect to the Walsh-Kaczmarz system, Analysis Math., 7 (1981), 141-150.

[16] A. A. S̆NEIDER, On series with respect to the Walsh functions with monotone coefficients, Izv. Akad. Nauk SSSR Ser. Math., 12 (1948), 179-192.

[17] O. SZÁsz, On the logarithmic means of rearranged partial sums of a Fourier series, Bull. Amer. Math. Soc., 48 (1942), 705-711.

[18] K. YABUTA, Quasi-Tauberian theorems, applied to the summability of Fourier series by Riesz's logarithmic means, Tohoku Math. J., II. Ser., 22 (1970), 117-129. 